Research Paper

\title{
Tropomyosin-1 Functions as a Tumor Suppressor with Respect to Cell Proliferation, Angiogenesis and Metastasis in Renal Cell Carcinoma
}

\author{
Jin Wang*, Chao Tang*, Chao Yang, Qi Zheng, Yuchuan Hou ${ }^{凶}$ \\ Department of Urology, the First Hospital of Jilin University, 71 Xinmin Street, Changchun 130021, Jilin, China. \\ *These authors contributed equally to this work. \\ $\triangle$ Corresponding author: Yuchuan Hou, Department of Urology, the First Hospital of Jilin University, 71 Xinmin Street, Changchun 130021, Jilin, China. Tel: \\ +86-0431-81875808; Fax: +86-0431-85654528; E-mail: hou63@163.com. \\ (C) Ivyspring International Publisher. This is an open access article distributed under the terms of the Creative Commons Attribution (CC BY-NC) license \\ (https://creativecommons.org/licenses/by-nc/4.0/). See http://ivyspring.com/terms for full terms and conditions.
}

Received: 2018.07.02; Accepted: 2019.03.31; Published: 2019.05.21

\begin{abstract}
Background: Tropomyosin-1 (TPMI) has long been known to be an actin-binding cytoskeletal protein. Multiple recent studies have revealed that TPMI is down-regulated in various malignant tumors, including renal cell carcinoma (RCC).

Methods: To further verify its role in RCC, transfection of a reconstructed plasmid was used to bi-directionally regulate TPMI levels. A colony formation assay, tube formation assay and invasion assay were adopted to assess cell proliferation, angiogenesis and metastasis, respectively, in the 786-O and ACHN cell lines. The xenograft tumor sizes were measured, and the microvessel density (MVD) was quantified. Western blot and immunohistochemistry (IHC) were used to detect key proteins involved in these processes.

Results: The colony formation assay and xenograft tumor models illustrated that TPMI up-regulation inhibited RCC cell proliferation. The tube formation assay and detection of vascular endothelial growth factor (VEGF) and cluster of differentiation 34 (CD34) in xenografts revealed that TPMI up-regulation inhibited angiogenesis in RCC. The invasion assay and detection of the E-cadherin and matrix metalloproteinases 9 (MMP-9) levels in xenografts demonstrated that TPM1 up-regulation inhibited tumor metastasis in RCC. Opposing effects were absent in TPMI down-regulation models.

Conclusions: TPMI functions as a tumor suppressor with respect to cell proliferation, angiogenesis and metastasis in RCC, suggesting that it is a potential therapeutic target for advanced RCC.
\end{abstract}

Key words: Renal cell carcinoma, Tropomyosin-1, Cell Proliferation, Angiogenesis, Metastasis

\section{Introduction}

Renal cell carcinoma (RCC) accounts for approximately $2 \%$ of all malignant tumors in adults, and clear cell renal cell carcinoma (ccRCC) is the predominant pathological subtype. In 2016, 62,700 new cases were diagnosed as kidney and renal pelvic cancer and 14,240 related deaths were reported in the US [1]. Chinese data show that there were 67,100 newly diagnosed kidney cancers and 24,100 related deaths in 2013 [2]. The overall 5-year survival rate for RCC has improved from $50 \%$ to $74 \%$ over the past three decades [1]. This improvement is primarily attributed to the growing proportion of early diagnosis of RCC, which is facilitated by the increasing use of medical imaging. While early detection has improved in this neoplasm, treatment of advanced or metastatic RCC remains limited. Since 
2005, clinical treatment of RCC has transitioned from a nonspecific immune approach to targeted therapy against vascular endothelial growth factor (VEGF) and now to novel immunotherapy agents [3]. Currently, mainstream RCC targeted therapies include tyrosine kinase inhibitors (TKIs) and mammalian target of rapamycin (mTOR) inhibitors, which are based on the inactivation of the tumor suppressor von Hippel-Lindau (VHL) and regulation of the hypoxia-inducible transcription factor (HIF)-VEGF pathway in most ccRCC. Although these agents have improved progression free survival, development of resistance is nearly universal, even for the new checkpoint inhibitors [4]. Therefore, identification of novel therapeutic targets is necessary to combat the resistance observed in classic target therapies.

Tropomyosin-1 (TPM1) is a tropomyosin family member that is known to be an actin-binding cytoskeletal protein in various cells. Tropomyosins function within the troponin complex to regulate muscle contraction in a calcium-dependent manner in muscle cells, while they have increased functional complexity in non-muscle cells and primarily act as microfilaments to stabilize the cytoskeleton [5]. Mutations in tropomyosins are directly involved in cardiac and skeletal muscle diseases, while alterations in tropomyosin expression directly contribute to the growth and spread of cancer [6]. Prasad and colleagues first reported that TPM1 is down-regulated and functions as a tumor suppressor gene (TSG) in breast cancer [7]. Subsequently, similar phenomena were observed in additional cancers, such as glioma, cholangiocarcinoma, oral squamous cell carcinoma and so on [8-10]. miRNA-21 is a well-known oncogene that is over-expressed in many tumor tissues compared with matched normal tissues. Suppression of miRNA-21 inhibits tumor growth through up-regulation of its target gene TPM1 [11]. TPM1 over-expression has been reported to stabilize F-actin and increase the number of cell-cell junctions, reducing the migratory potential of endothelial cells [12].

Our previous study demonstrated that TPM1 is down-regulated in both RCC patient tumors and cell lines. In vitro assays demonstrated that TPM1 is associated with RCC cell apoptosis, invasion and migration [13]. More recently, we reported that TPM1 promotes apoptosis via the p53-mediated mitochondrial pathway in RCC [14]. However, many details concerning the correlation of TPM1 and RCC cell proliferation and angiogenesis have yet to be reported, and the role of TPM1 as a tumor suppressor in RCC is still not fully established. The present study aimed to further investigate the correlations between
TPM1 regulation and cell proliferation, angiogenesis, and metastasis in RCC. We assessed its tumor suppressive effects using bi-directional regulation of TPM1 both in vitro and in vivo using two RCC cell lines.

\section{Materials and methods}

\section{Cell culture and antibodies}

The 786-O and ACHN cell lines were both obtained from Procell Life Science \& Technology (Wuhan, China) and maintained in RPMI medium and MEM medium (Gibco, Rockville, MD, USA), respectively. Cells were also cultured with $10 \%$ fetal bovine serum (Hyclone, Rockville, MD, USA) and 1\% penicillin/streptomycin in $5 \times 7 \mathrm{~cm}$ flasks. Cells were kept in a $37^{\circ} \mathrm{C}$ incubator in a $5 \% \mathrm{CO}_{2}$ fully humidified atmosphere. The anti-tropomyosin-1 antibody was obtained from Cell Signaling Technology (Danvers, MA, USA), while the anti-MMP-9, anti-VEGF, anti-E-cadherin, anti-CD34 and anti- $\beta$-actin antibodies were purchased from Biosynthesis Biotechnology (Beijing, China).

\section{Plasmid construction and transient transfection}

We purchased reconstructed plasmids from Suzhou GenePharma (Suzhou, China), including the over expression (OE) plasmid pEX4-TPM1, over-expression negative control (OE-NC) plasmid pEX4-NC, knock-down (KD) plasmid pGPU6/GFP/ Neo-shTPM1 and knock-down negative control (KD-NC) plasmid pGPU6/GFP/Neo-shNC. In addition to these four groups, we designated another Mock group using Lipofectamine ${ }^{\mathrm{TM}} 2000$ only with no vector. 786-O and ACHN cells were seeded into 24-well microplates (approximately $4 \times 10^{4}$ cells/well) and cultured for 24 to 48 hours. We transfected both cell lines with the plasmids mentioned above when cell confluence reached approximately $70 \%-80 \%$ using Lipofectamine $^{\mathrm{TM}} 2000$ (Invitrogen, Carlsbad, CA, USA) according to the manufacturer's instructions. The ratio of plasmid to transfection reagent was 1:1.5.

\section{Colony formation assay}

Twenty-four hours after transfection, 786-O and ACHN cells were seeded into 6-well plates at approximately 500 cells per well. Cells were cultured for 8 to 10 days until small colonies could be clearly seen. Plates containing colonies were gently washed with PBS and fixed with ethanol for 10 minutes. Wells were rinsed again with PBS and the colonies were stained with $0.1 \%$ crystal violet solution in ethanol for 10 minutes. Experiments were performed three times in duplicate. 


\section{Tube formation assay}

Matrigel $^{\mathrm{TM}}$ (BD Biosciences, San Jose, CA, USA) was kept at $4^{\circ} \mathrm{C}$ overnight for precooling, and then, 60 $\mu \mathrm{l}$ of Matrigel ${ }^{\mathrm{TM}}$ was transferred to a 96-well plate. Twenty-four hours after transfection, ACHN and 786-O cells were collected, diluted and transferred to the assigned wells in a 96-well plate $\left(2 \times 10^{4}\right.$ cells / well). After 8 hours of incubation, tube formation was imaged using a microscope at $40 \times$ magnification and quantified by counting the branch points in 3 randomly selected microscopic fields per well. Experiments were performed twice in duplicate.

\section{Invasion assay}

The invasive behaviors of 786-O and ACHN cells in response to alterations in TPM1 expression were assessed using Matrigel ${ }^{\mathrm{TM}}$ (BD Biosciences, San Jose, CA, USA) precoated 24-well Transwell ${ }^{\circledR}$ plates (Corning Costar, Corning, NY, USA). Twenty-four hours after transfection, we added $100 \mu \mathrm{l}\left(10^{6} \mathrm{cell} / \mathrm{ml}\right)$ of the cell suspension into the upper chamber and higher serum concentration medium into the lower chamber. After incubation for another 36 hours, cells that invaded to the bottom of the inserts were fixed in $2 \%$ paraformaldehyde in methanol and dyed with a $0.1 \%$ crystal violet solution. Three random 100× magnification microscopic fields were selected for cell counting.

\section{Xenograft tumor models}

Five groups of three mice each were subcutaneously injected with $100 \mu$ l (approximately $10^{7}$ cells) of the ACHN cell suspension in the scapular region. ACHN cells were transfected with five different plasmids 24 hours prior to injection. The tumor size was measured via calipers at the injection site every six days. The tumor volume was calculated using the formula $0.5 \mathrm{ab}^{2}$, where $\mathrm{a}$ and $\mathrm{b}$ represent the larger and smaller of the 2 diameters, respectively. Four weeks later, mice were injected intraperitoneally with pentobarbital sodium $200 \mathrm{mg} / \mathrm{kg}$ for euthanasia. Immunodeficient mice $(\mathrm{BALB} / \mathrm{c})$ were purchased from Beijing Vital River Laboratory Animal Technology (Beijing, China). All experimental protocols were approved by the animal experiments ethical committee of Jilin University, and nude mice were cared for and manipulated in accordance with the committee's guidelines. All possible methods were utilized to reduce animal suffering while maintaining a certain number of animals in the study.

\section{Western blotting}

After nude mice were sacrificed, tumor specimens were immediately harvested and immersed in liquid nitrogen. One hundred milligrams of tumor tissue with $1 \mathrm{ml}$ of RIPA lysis buffer suspended via a homogenizer. For 786-O and ACHN cell lines, both were harvested 36 hours after transfection. Cells were suspended in ice-cold buffer and homogenized after centrifugation. Homogenates were centrifuged at $10,000 \mathrm{~g}$ at $4^{\circ} \mathrm{C}$ for 10 minutes to obtain supernatants. Equal amounts of denatured protein were loaded on $10 \%$ polyacrylamide gels and separated by electrophoresis. Specific proteins were detected by immunoblotting using anti-TPM1 (1:1000), anti-VEGF (1:500), anti-E-cadherin (1:1000) and anti- $\beta$-actin (1:1000) antibodies.

\section{Immunohistochemistry (IHC) staining}

Xenograft tumor tissues were fixed for 36 hours in paraformaldehyde followed by embedding in paraffin wax. Tissue blocks were cut into thin slices and dried for 35 minutes at $60^{\circ} \mathrm{C}$ in an oven. Slides were deparaffinized with xylene and rehydrated through a series of graded ethanol. Antigen retrieval was performed in $10 \mathrm{mM}$ sodium citrate buffer for 20 minutes in a microwave. Endogenous peroxidase was eliminated by a hydrogen peroxide solution, and slides were blocked with 5\% BSA. Anti-MMP-9 (1:500) and anti-CD34 (1:500) primary antibodies were applied for overnight incubation at $4^{\circ} \mathrm{C}$, followed by incubation with secondary antibody conjugated to streptavidin-horseradish peroxidase (Maixin biotechnology, Fuzhou, China). The immunoreactive score (IRS) was calculated as previously reported [13].

\section{Statistical analysis}

All statistical analyses were performed using GraphPad Prism 5.0 software (GraphPad Software, Inc., La Jolla, CA, USA). All data are expressed as the mean \pm standard deviation unless otherwise stated. One-way or two-way analysis of variance (ANOVA) followed by the Bonferroni test was used for comparisons between groups. $\mathrm{P}<0.05$ was considered statistically significant.

\section{Results}

\section{TPM1 suppresses cell proliferation and tumorigenesis in RCC}

Transient transfection of pEX4-TPM1 and pGPU6-shTPM1 in 786-O and ACHN cell lines was utilized to investigate the effects of TPM1 on RCC progression. The expression levels of TPM1 protein were detected by western blotting 24 hours after transfection. Both reconstructed plasmids were successfully transfected into the 786-O and ACHN cell lines. pEX4-TPM1 over-expression plasmid worked as expected while pGPU6-shTPM1 knock-down plasmid did not. These data were reported in our previous published article. 

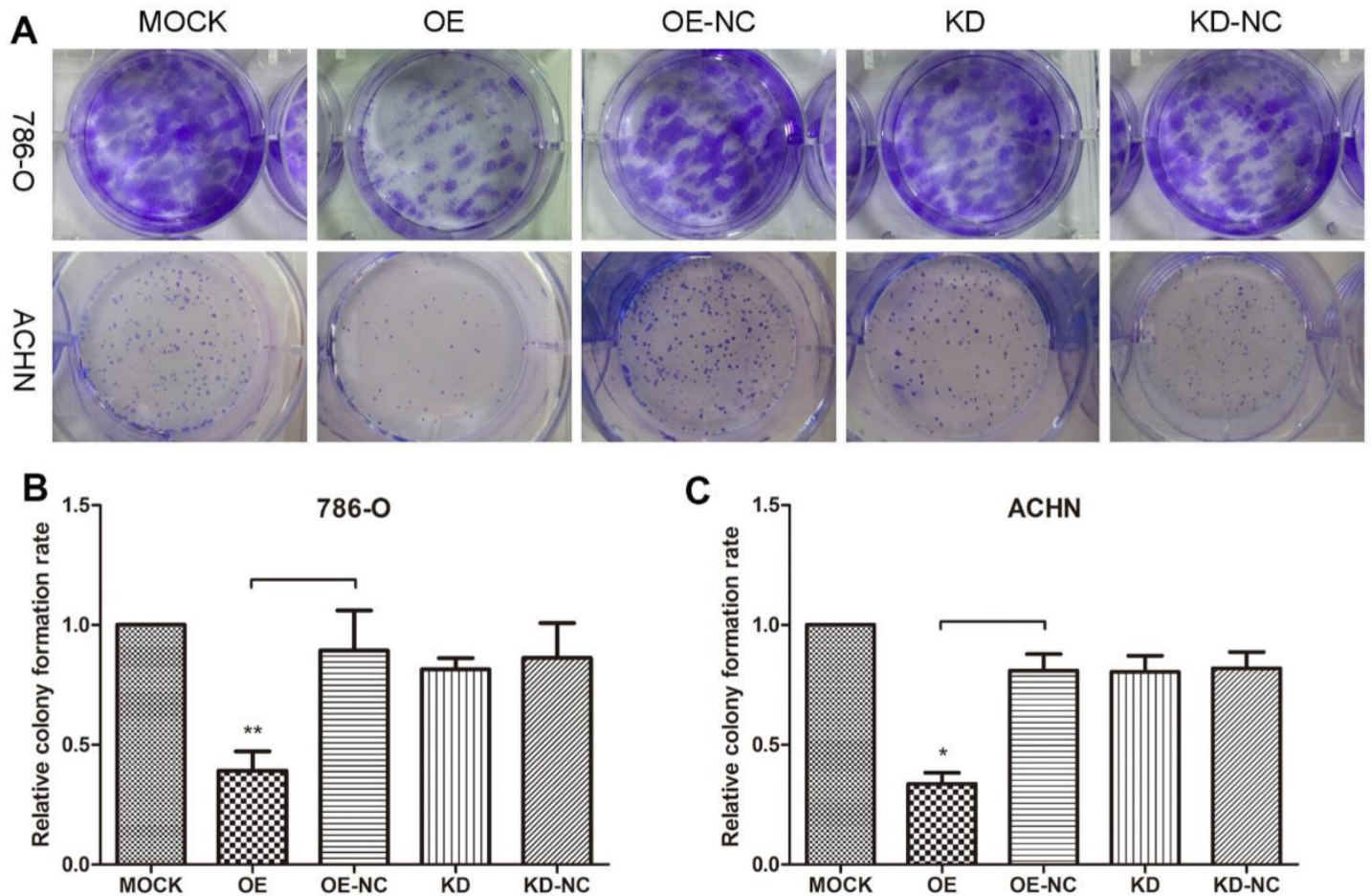

Figure 1. TPMI up-regulation inhibits cell proliferation in 786-O and ACHN cell lines. (A) Representative pictures of colonies formed 10 days after plasmid transfection from each group. Relative colony formation rates of different groups in $786-\mathrm{O}$ cells (B) and ACHN cells (C). Colony formation rate of the Mock group was defined as $100 \%$ for both cell lines. Values indicated by bars are mean \pm standard deviation of 3 experimental repeats. ${ }^{*}<0.05, * * p<0.01$ and $* * * p<0.001$, compared with assigned negative control groups.

Colony formation assays were performed to evaluate 786-O and ACHN cell growth changes in response to changes in the TPM1 levels (Figure 1A). The results revealed remarkable reductions in colonies formed in the pEX4-TPM1 over-expression groups compared to the $\mathrm{pEX} 4-\mathrm{NC}$ negative controls (Figure $1 \mathrm{~B}$ and 1C). No obvious difference was observed between the TPM1 knock-down groups and knock-down negative control groups (Figure 1B and 1C).

We utilized xenograft animal models to further assess the role of TPM1 in tumorigenesis. Compared to the negative controls, tumor burden was significantly reduced in response to TPM1 over-expression and was nearly unchanged in the TPM1 knock-down groups (Figure 2A). The OE group exhibited significant reductions in the average tumor volume from the beginning to the end of the experiments as well as delayed tumor formation compared to its counterpart, the OE-NC group (Figure 2B). Opposing phenomena were absent when we determined the tumor volumes in the $\mathrm{KD}$ and KD-NC groups (Figure 2C). Our results indicate that TPM1 suppresses RCC cell proliferation and tumorigenesis both in vitro and in vivo.

\section{TPM1 inhibits RCC angiogenesis in vitro and in vivo}

We performed a tube formation assay to profile the effects of TPM1 on angiogenesis in RCC. The branch points in each microscopic field, which represent the density of newly formed vessels, were significantly reduced in the TPM1 OE groups compared with their counterparts, the OE-NC groups (Figure 3A and 3B). The branch points in the TPM1 $\mathrm{KD}$ group were significantly increased compared to those in its counterpart, the KD-NC group, in 786-O cells, while this difference was absent in ACHN cells (Figure 3A and 3B).

Proteins were extracted from RCC tumor tissues, and western blotting was used to detect expression changes in VEGF in response to changes in TPM1 expression. The western blot results demonstrated that the TPM1 levels in xenograft tumors were significantly up-regulated after pEX4-TPM1 transfection but remained unchanged after pGPU6-shTPM1 transfection compared to those in their counterpart negative control groups (Figure 4A and 4B). The VEGF bands indicated that TPM1 up-regulation significantly decreases its expression levels (Figure 4A and 4B). VEGF expression showed no obvious difference between the TPM1 KD and KD-NC groups (Figure 4A and 4B). To further verify the anti-angiogenic effect of TPM1 in vivo, xenograft tumors from nude mice were sectioned and stained with the endothelial cell marker CD34. The CD34 staining results revealed that the microvessel density (MVD) was drastically reduced in the TPM1 OE group compared to that in the OE-NC group (Figure $4 \mathrm{C}$ and $4 \mathrm{D})$. Similar to previous results, this difference 
was absent between the $\mathrm{KD}$ and $\mathrm{KD}-\mathrm{NC}$ groups (Figure 4C and 4D).

\section{TPM1 restrains invasion by regulating E-cadherin and MMP-9 in RCC}

As a cytoskeleton protein, TPM1 might participate in RCC cell motility. A Transwell ${ }^{\circledR}$ invasion assay was performed to evaluate invading

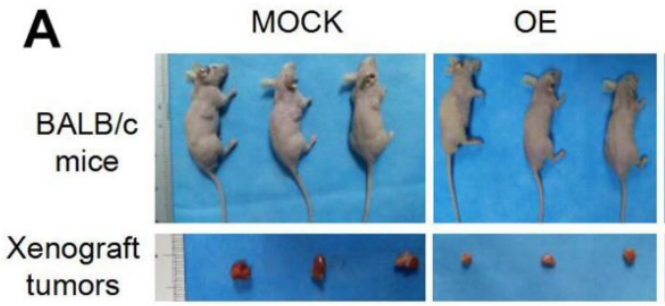

B

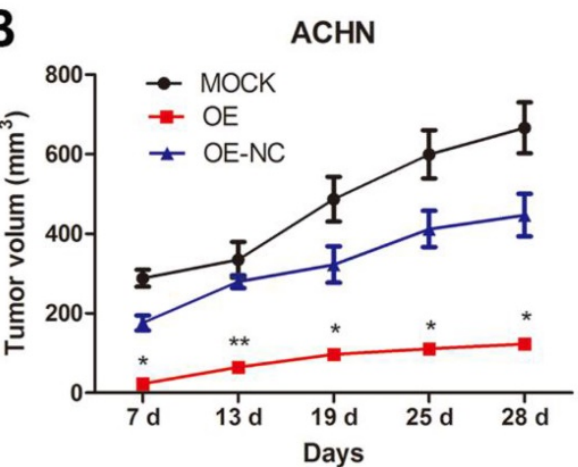

cells 60 hours after transfection with the different plasmids. Compared with the negative control groups, TPM1 over-expression clearly restrained the invasion ability of both RCC cell lines (Figure 5A and $5 B)$. However, the opposite effects were not observed in response to down-regulation of TPM1 in RCC cells (Figure 5A and 5B).
OE-NC

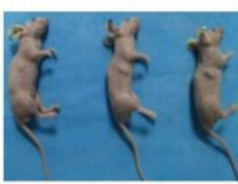

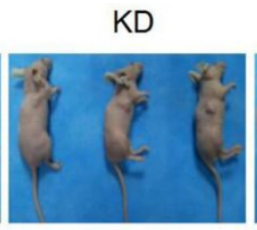

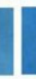

C

ACHN

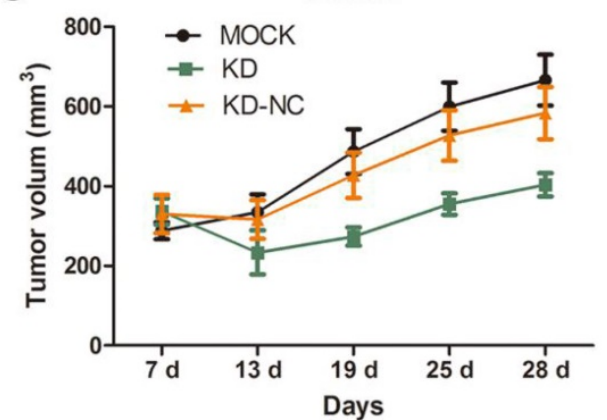

Figure 2. TPM1 suppresses RCC tumorigenesis and tumor growth in xenograft animal models. (A) Representative images showing xenograft tumors in nude mice and resected tumors from different treatment groups. (B-C) Tumor growth curves of different groups. (OE and OE-NC groups (B); KD and KD-NC groups (C)). Average tumor size of 3 mice per group is presented. Values indicated by bars are mean \pm standard deviation. ${ }^{*} \mathrm{p}<0.05,{ }^{* *} \mathrm{p}<0.01$ and $* * * \mathrm{p}<0.001$, compared with assigned negative control groups.

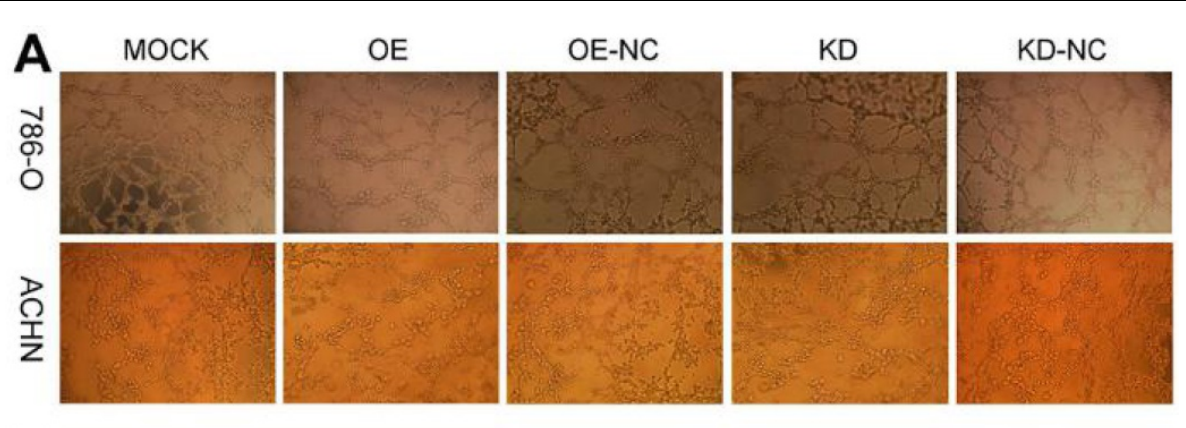

B

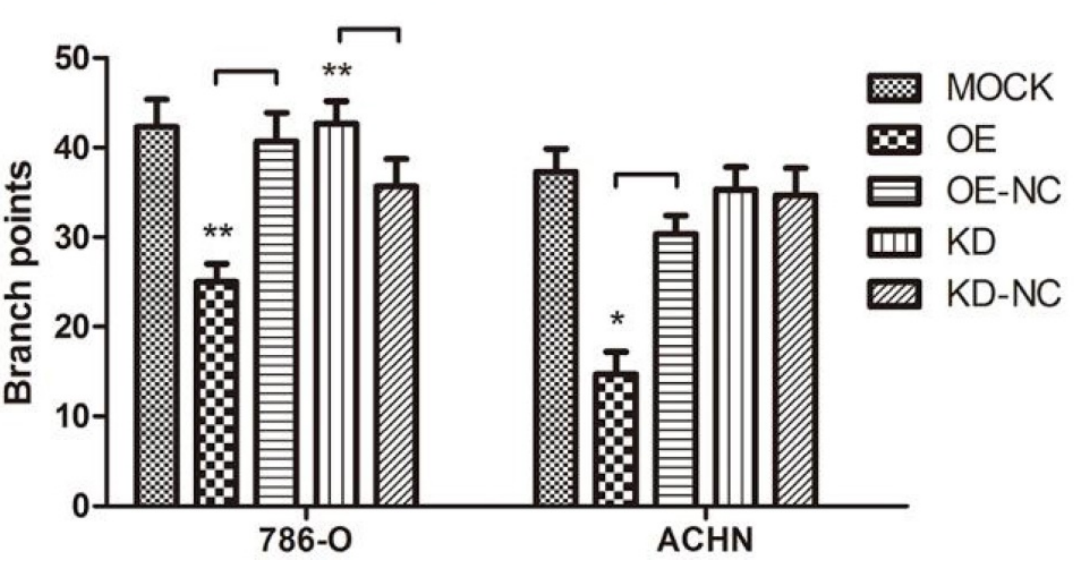

Figure 3. TPM1 up-regulation suppresses angiogenesis in 786-O and $\mathrm{ACHN}$ cell lines. (A) Representative images under phase contrast microscopy of tubes formed 8 hours after incubation with Matrigel ${ }^{\mathrm{TM}}$ in two RCC cell lines. (B) Statistical analysis of number of branch points for different groups in 786-O cells and ACHN cells. Reduced from $\times 40$. Values indicated by bars are mean \pm standard deviation of 3 randomly selected microscopic fields. $* p<0.05$, ** $p<0.01$ and $* * * p<0.001$, compared to negative control groups. 

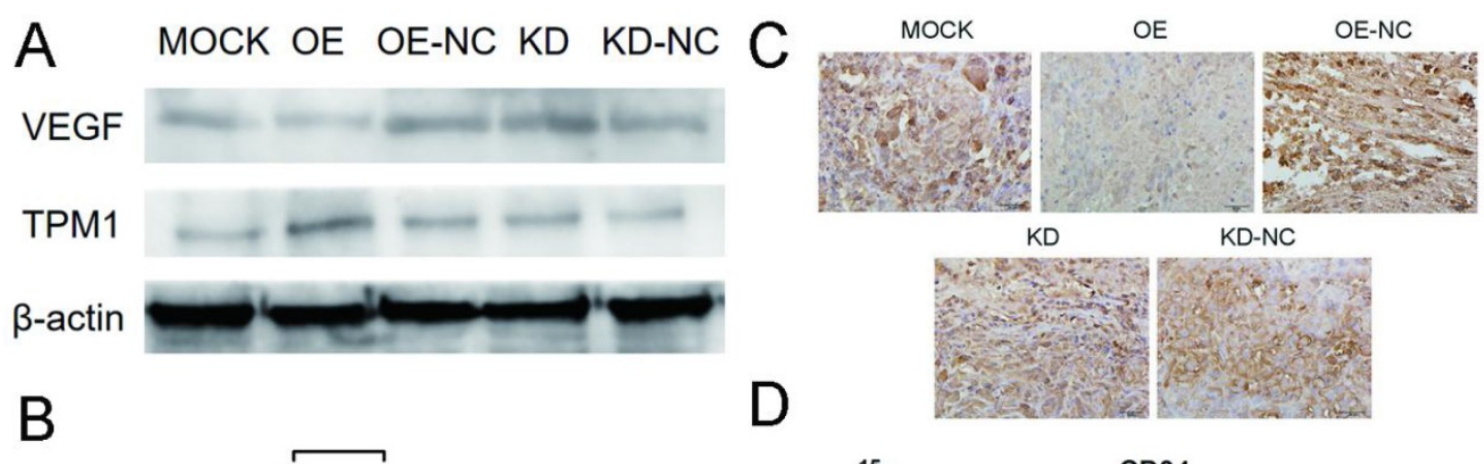

KD KD-NC
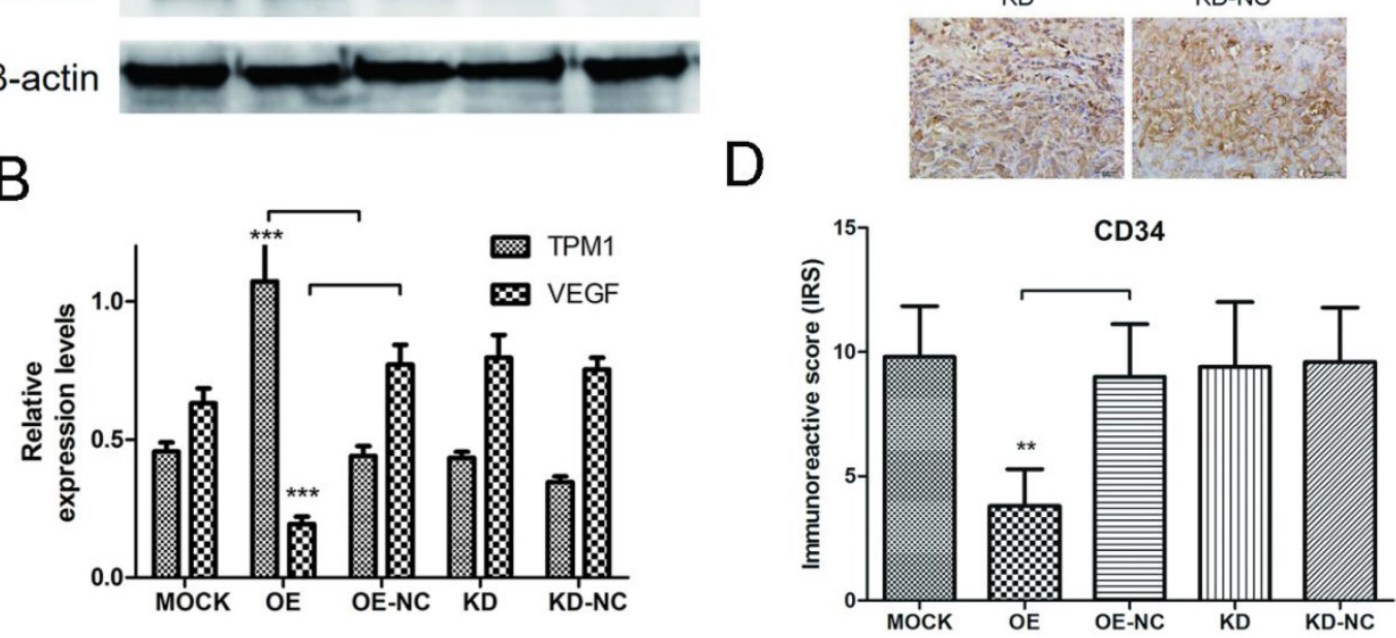

Figure 4. TPMI suppresses RCC angiogenesis by down-regulating VEGF expression in vivo. (A) Representative cropped protein bands of VEGF, TPMI and $\beta$-actin from different treatment groups. (B) Relative expression levels of VEGF were significantly down-regulated in response to TPMI over-expression. (C) Representative IHC staining for CD34 in xenograft tumor sections from different treatment groups. (D) Immunoreactive scores of CD34 staining demonstrated that xenograft tumor MVD decreased significantly in response to TPMI up-regulation. Reduced from $\times 400$. Values indicated by bars are mean \pm standard deviation of 3 repeats. * $p<0.05$, $* * p<0.01$ and $* * * p<0.001$, compared to negative control groups.
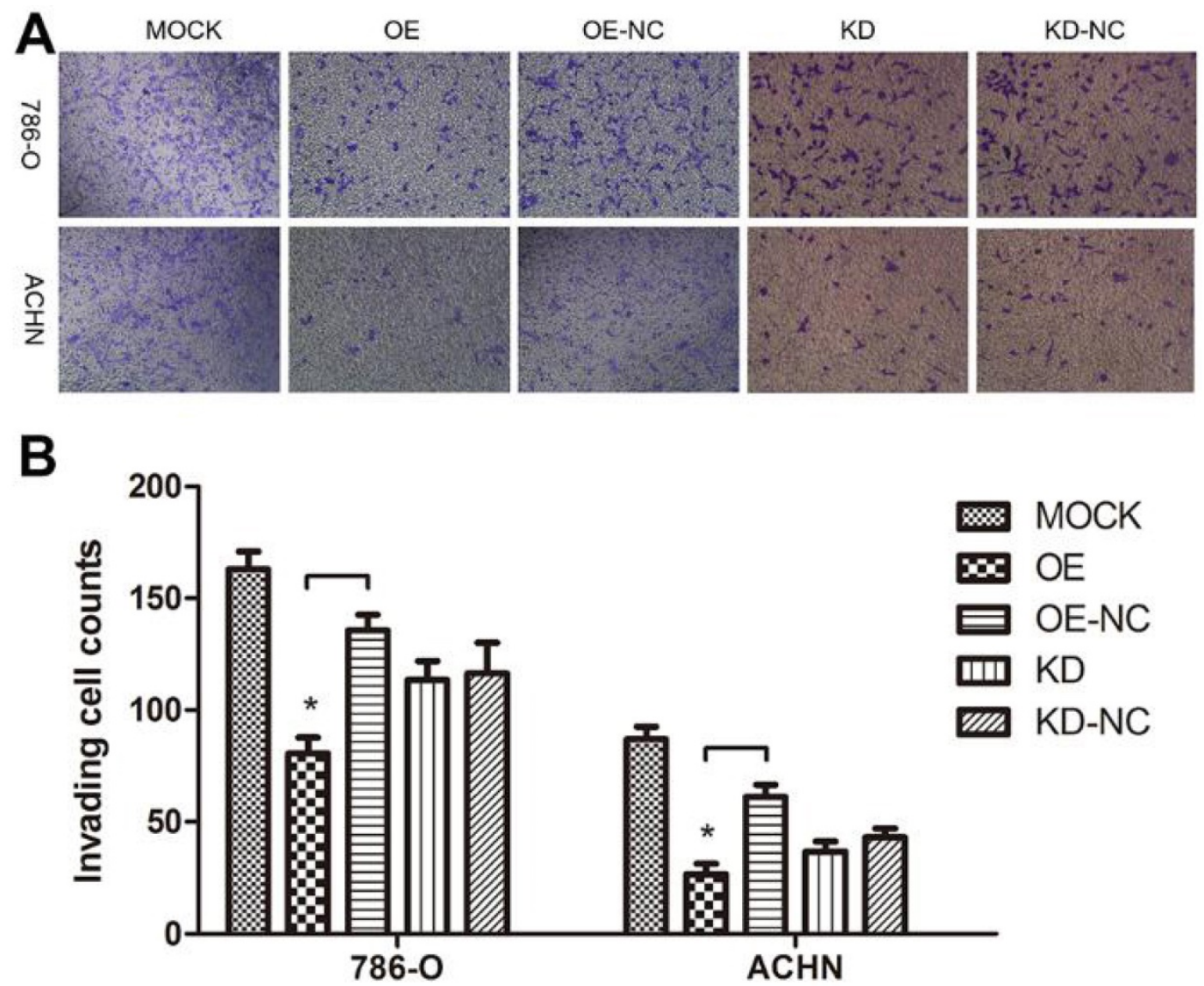

Figure 5. TPMI up-regulation inhibits cell motility in 786-O and ACHN cell lines. (A) Invading cells through the Transwell membrane were stained and imaged for different treatment groups. (B) Statistical analysis of invading cell number for different groups in 786-O cells and ACHN cells. Reduced from $\times 100$. Values indicated by bars are mean \pm standard deviation of cell numbers in three randomly selected microscopic fields. ${ }^{*} \mathrm{p}<0.05$, $* * \mathrm{p}<0.01$ and $* * * \mathrm{p}<0.001$, compared to negative control groups. 

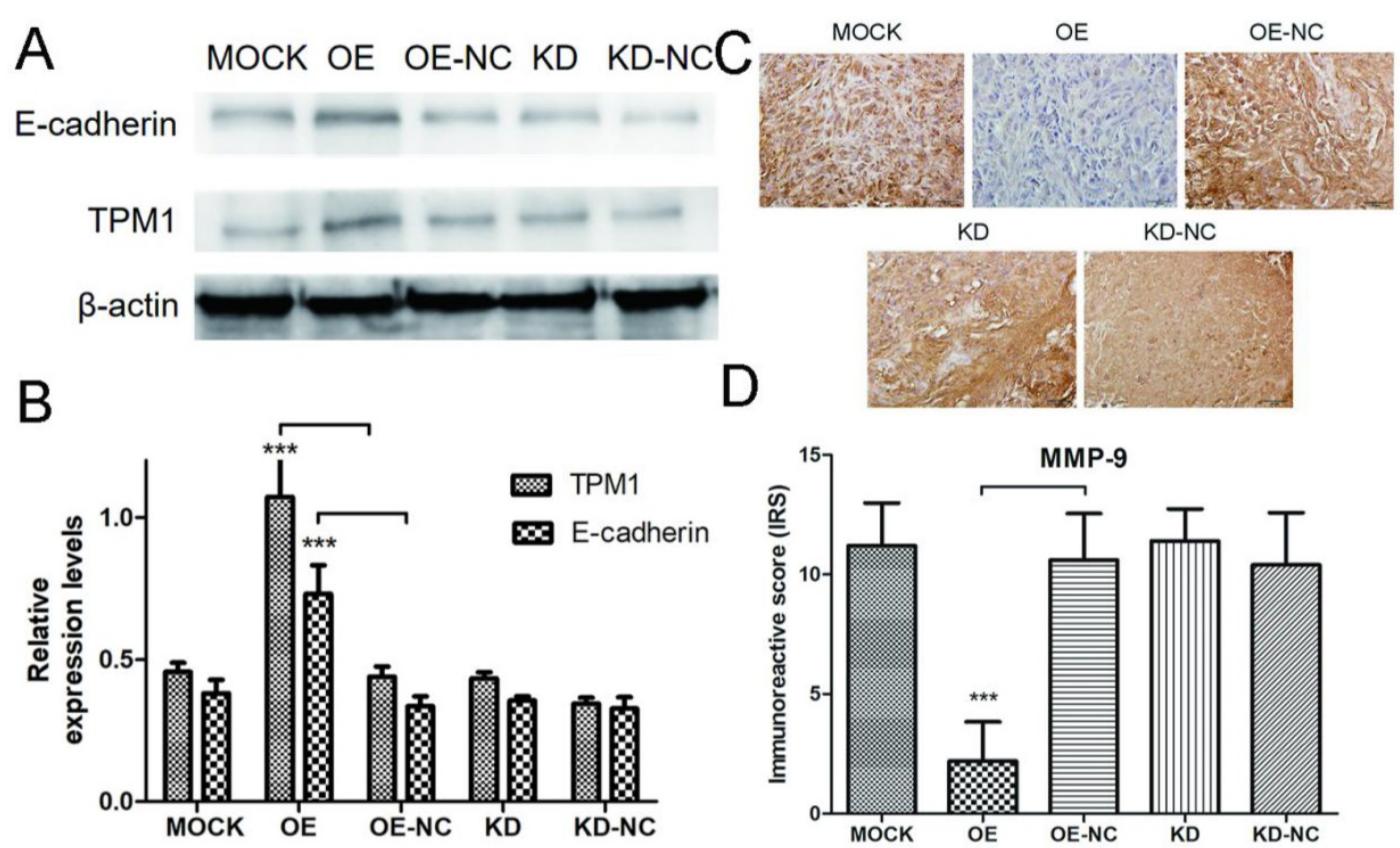

Figure 6. TMPI over-expression correlates with increased expression of E-cadherin and decreased expression of MMP-9 in vivo. (A) Representative cropped protein bands of E-cadherin, TPMI and $\beta$-actin from different treatment groups. (B) Relative expression levels of E-cadherin were significantly up-regulated in response to TPMI over-expression. (C) Representative IHC staining for MMP-9 in xenograft tumor sections from different treatment groups. (D) Immunoreactive scores of MMP-9 staining demonstrated that MMP-9 was significantly down-regulated by TPM1 over-expression. Reduced from $\times 400$. Values indicated by bars are mean \pm standard deviation of three experimental repeats. $* p<0.05, * * p<0.01$ and $* * * p<0.001$, compared to negative control groups.

Next, we performed western blotting and IHC staining on xenograft tumors to explore the potential mechanisms of TPM1-mediated inhibition of RCC metastasis. The expression levels of E-cadherin and MMP-9 were measured by western blotting and IHC in response to changes in TPM1 expression. The results demonstrated that the E-cadherin expression levels markedly increased and the MMP-9 expression levels significantly decreased in response to TPM1 up-regulation in vivo (Figure 6A-6D). Similar to our other observations, no changes in E-cadherin or MMP-9 expression were observed in the TPM1 KD group compared with the KD-NC group (Figure $6 \mathrm{~A}-6 \mathrm{D})$.

\section{Discussion}

The most important characteristics of malignant tumors are uncontrolled cell proliferation and infinite tumor growth. This study illustrated that TPM1 suppresses cell proliferation in vitro and inhibits tumor growth in vivo. These results are consistent with our previous observations from the MTT assay [14]. It has been reported that miRNA-21 is over-expressed in RCC tissue and modulates growth, apoptosis and cell cycle progression of RCC cells and regulates expression of programmed cell death 4 (PDCD4) and TPM1 [15]. TPM1 modulates all of these tumor suppressing effects, except for the cell cycle, according to our results. Other miRNAs, such as miRNA-107, also target TPM1 to influence cell proliferation and other tumor behaviors [16].

With the rapid growth of malignant tumors, hypoxia stress occurs inside the tumor, initiating multiple angiogenesis signaling pathways to meet the increasing demand for oxygen. Many anti-tumor drugs have been developed based on this theory. Prior to our study, TPM1 was found to be an intermediary in glioma between 4'-acetoamido-4hydroxychalcone (AHC) and its anti-tumor effects, which include suppressing angiogenesis [8]. TPM1 is regulated by hypoxia to participate in angiogenesis-mediated cytoskeletal remodeling in human umbilical vein endothelial cells (HUVECs) [17]. By contrast, hyperoxia also down-regulates expression of TPM1 in neural crest stem cells [18]. Our study demonstrated that TPM1 up-regulation inhibits RCC angiogenesis by decreasing VEGF expression. Targeting angiogenic factors from the VEGF family has become an effective strategy for inhibiting tumor growth in RCC [19]. We hypothesize that TPM1 may be another potential target from the VEGF pathway for the treatment of advanced RCC.

Another characteristic of malignant tumors is metastasis. miRNA-21 targets TPM1, PDCD4 and maspin to regulate invasion and metastasis in breast cancer cells [20]. As mentioned above, TPM1 has been reported to stabilize cell-cell junctions and reduce the migratory potential of endothelial cells [12]. Matrix metalloproteinases (MMPs) and other extracellular matrix-degrading enzymes participate in RCC invasion and metastasis. Epithelial-mesenchymal 
transition (EMT) transcription factors, such as Snail and Slug, promote tumor cell invasion through up-regulation of MMPs and down-regulation of E-cadherin [21]. Thus, we hypothesized that TPM1 suppresses RCC cell mobility through regulation of those molecules. The transwell assay results confirmed the role of TPM1 in attenuating cell invasion. Further observation demonstrated that TPM1 over-expression correlated with increases in E-cadherin expression and decreases in MMP-9 expression. It can be inferred that TPM1 is involved in EMT, a key process in tumor invasion and distant dissemination. E-cadherin, MMP-9 and even VEGF participate in cell adherence and EMT [22].

In addition to cell proliferation, angiogenesis and metastasis, we previously reported that TPM1 is down-regulated in RCC and could induce RCC cell apoptosis $[13,14]$. Other studies found that decreased TPM1 might be one mechanism underlying radiation resistance and also a potential target for overcoming it in glioma [23]. Based on our findings in RCC and those of many other researchers in other tumors, it can be concluded that TPM1 is a novel TSG in RCC.

The highest impact of discovering a novel TSG in cancer is the opportunity to develop a new treatment method or to overcome resistance to targeted therapy. Prior to 2005, medical therapies for RCC were limited to cytokine therapies, which are very toxic and benefit only a small percentage of patients. As of 2017, therapeutic agents also include kinase and immune checkpoint inhibitors [24]. Inhibition of the VHL/ HIF/ VEGF and PI3K/ AKT/ mTOR pathways decrease protein translation and inhibit both angiogenesis and tumor cell proliferation. Although TKIs are the primary first-line treatment option for advanced RCC, all patients eventually become resistant to TKIs [25]. To overcome this resistance, second-line treatments based on different mechanisms can be used, including mTOR inhibitors (mTORi), programmed death 1 (PD-1) inhibitors, programmed death ligand 1 (PD-L1) inhibitors, or various combinations among them. TPM1 is reported to be related to the MEK/ERK and NF-KB signaling pathways in osteosarcoma [16]. Thus, TPM1 may be utilized to prevent tumor angiogenesis and apoptosis escape when RCC become resistant to TKI and mTORi.

In summary, this study revealed that TPM1 functions as a tumor suppressor primarily by inhibiting cell proliferation, angiogenesis and metastasis in RCC. This observation corroborated our hypothesis based on our previous studies. TPM1 is a potential therapeutic target for advanced RCC.

\section{Abbreviations}

TPM1: tropomyosin-1; RCC: renal cell carcinoma; MVD: microvessel density; IHC: immunohistochemistry; VEGF: vascular endothelial growth factor; CD34: cluster of differentiation 34; MMP-9: matrix metalloproteinases 9; ccRCC: clear cell renal cell carcinoma; TKIs: tyrosine kinase inhibitors; mTORi: mammalian target of rapamycin inhibitors; VHL: von Hippel-Lindau; HIF: hypoxia-inducible transcription factor; TSG: tumor suppressor gene; OE: over expression; OE-NC: over-expression negative control; KD: knock-down; KD-NC: knock-down negative control; ANOVA: analysis of variance; PDCD4: programmed cell death 4; AHC: 4'-acetoamido-4-hydroxychalcone; HUVECs: human umbilical vein endothelial cells; EMT: epithelialmesenchymal transition; PD-1: programmed death 1; PD-L1: programmed death ligand 1.

\section{Acknowledgements}

This work was supported by the $8^{\text {th }}$ Youth Development Program of the First Hospital, Jilin University (grant number JDYY82017015).

\section{Author Contributions}

Conception and design of experiments: Jin Wang. Implementation of experiments: Chao Tang. Data collection: Chao Tang and Chao Yang. Data analysis and visualization: Jin Wang and Chao Yang. Writing original drafts: Chao Tang and Qi Zheng. Review and modification: Jin Wang and Yuchuan Hou. Supervision: Jin Wang and Yuchuan Hou. Funding acquisition: Yuchuan Hou.

\section{Availability of data and materials}

All relevant data of the study were already included in the manuscript.

\section{Ethics approval and consent}

This study was approved by the Ethics Committee of the First Hospital, Jilin University. Animal experiments were conducted according to the guidelines and rules.

\section{Competing Interests}

The authors have declared that no competing interest exists.

\section{References}

1. Siegel RL, Miller KD, Jemal A. Cancer statistics, 2016. CA Cancer J Clin. 2016; 66(1): 7-30.

2. Chen $\mathrm{W}$, Zheng R, Zhang $\mathrm{S}$, et al. Cancer incidence and mortality in China, 2013. Cancer Lett. 2017; 401: 63-71.

3. Barata PC, Rini BI. Treatment of renal cell carcinoma: Current status and future directions. CA Cancer J Clin. 2017; 67(6): 507-524.

4. Siska PJ, Beckermann KE, Rathmell WK, Haake SM. Strategies to overcome therapeutic resistance in renal cell carcinoma. Urol Oncol. 2017; 35(3): 102-110. 
5. Perry SV. Vertebrate tropomyosin: distribution, properties and function. J Muscle Res Cell Motil. 2001; 22(1): 5-49.

6. Gunning P, O'Neill G, Hardeman E. Tropomyosin-based regulation of the actin cytoskeleton in time and space. Physiol Rev. 2008; 88(1): 1-35.

7. Bharadwaj S, Prasad GL. Tropomyosin-1, a novel suppressor of cellular transformation is downregulated by promoter methylation in cancer cells. Cancer Lett. 2002; 183(2): 205-213.

8. Ku BM, Ryu HW, Lee YK, et al. 4 '-Acetoamido-4-hydroxychalcone, a chalcone derivative, inhibits glioma growth and invasion through regulation of the tropomyosin 1 gene. Biochem Biophys Res Commun. 2010; 402(3): 525-530.

9. Yang $W$, Wang $X$, Zheng $W$, et al. Genetic and epigenetic alterations are involved in the regulation of TPM1 in cholangiocarcinoma. Int J Oncol. 2013; 42(2): 690-698.

10. Pan H, Gu L, Liu B, et al. Tropomyosin-1 acts as a potential tumor suppressor in human oral squamous cell carcinoma. PLoS One. 2017; 12(2): e0168900.

11. Zhu SM, Si ML, Wu HL, Mo YY. MicroRNA-21 targets the tumor suppressor gene tropomyosin 1 (TPM1). J Biol Chem. 2007; 282(19): 14328-14336.

12. Gagat M, Grzanka D, Izdebska M, Grzanka A. Effect of L-homocysteine on endothelial cell-cell junctions following F-actin stabilization through tropomyosin-1 overexpression. Int J Mol Med. 2013; 32(1): 115-129.

13. Wang J, Guan J, Lu Z, et al. Clinical and tumor significance of tropomyosin-1 expression levels in renal cell carcinoma. Oncol Rep. 2015; 33(3): 1326-1334.

14. Tang C, Wang J, Wei Q, et al. Tropomyosin-1 promotes cancer cell apoptosis via p53-mediated mitochondrial pathway in renal cell carcinoma. Oncol Lett. 2018; 15(5): 7060-7068.

15. Lv L, Huang F, Mao H, et al. MicroRNA-21 is overexpressed in renal cell carcinoma. Int J Biol Markers. 2013; 28(2): 201-207.

16. Jiang R, Zhang C, Liu G, et al. MicroRNA-107 Promotes Proliferation, Migration, and Invasion of Osteosarcoma Cells by Targeting Tropomyosin 1. Oncol Res. 2017; 25(8): 1409-1419.

17. Weigand JE, Boeckel JN, Gellert P, Dimmeler S. Hypoxia-induced alternative splicing in endothelial cells. PLoS One. 2012; 7(8): e42697.

18. Chen CC, Hsia CW, Ho CW, et al. Hypoxia and Hyperoxia Differentially Control Proliferation of Rat Neural Crest Stem Cells via Distinct Regulatory Pathways of the HIF1 alpha-CXCR4 and TP53-TPM1 Proteins. Dev Dynam. 2017; 246(3): 162-185

19. Dimova I, Popivanov G, Djonov V. Angiogenesis in cancer - general pathways and their therapeutic implications. J Buon. 2014; 19(1): 15-21.

20. Zhu S, Wu H, Wu F, et al. MicroRNA-21 targets tumor suppressor genes in invasion and metastasis. Cell Res. 2008; 18(3): 350-359.

21. Mikami S, Oya M, Mizuno R, et al. Recent advances in renal cell carcinoma from a pathological point of view. Pathol Int. 2016; 66(9): 481-490.

22. Matoba R, Morizane Y, Shiode Y, et al. Suppressive effect of AMP-activated protein kinase on the epithelial-mesenchymal transition in retinal pigment epithelial cells. PLoS One. 2017; 12(7): e0181481.

23. Du HQ, Wang $Y$, Jiang $Y$, et al. Silencing of the TPM1 gene induces radioresistance of glioma U251 cells. Oncol Rep. 2015; 33(6): 2807-2814.

24. Posadas EM, Limvorasak S, Figlin RA. Targeted therapies for renal cell carcinoma. Nat Rev Nephrol. 2017; 13(8): 496-511.

25. Duran I, Lambea J, Maroto P, et al. Resistance to Targeted Therapies in Renal Cancer: The Importance of Changing the Mechanism of Action. Target Oncol. 2017; 12(1): 19-35. 